\title{
Repercusión en Twitter de las metodologías activas ABP, Flipped Classroom y Gamificación
}

\section{(Impact on Twitter of active methodologies PBL, flipped classroom and gamification)}

\author{
Rosabel Roig-Vila \\ Juan Francisco Álvarez Herrero \\ Universidad de Alicante (España)
}

DOI: $\underline{\text { http://dx.doi.org/10.5944/ried.22.2.23272 }}$

\section{Cómo referenciar este artículo:}

Roig-Vila, R., y Álvarez Herrero, J. F. (2019). Repercusión en Twitter de las metodologías activas ABP, Flipped Classroom y Gamificación. RIED. Revista Iberoamericana de Educación a Distancia, 22(2), pp. 79-96. doi: http://dx.doi. org/10.5944/ried.22.2.23272

\section{Resumen}

Twitter es una red social donde los usuarios interactúan en un espacio digital común, formando conexiones que, en sí, configuran, a su vez, redes propias de interacción a partir del intercambio de contenido. En este artículo, se ha abordado esta temática en torno a la repercusión que las metodologías activas tienen en Twitter. Para ello, se ha llevado a cabo una investigación donde se utilizó la técnica de análisis de redes sociales, tanto en el análisis del tipo de mensaje como su representación gráfica. Las metodologías activas analizadas fueron el Aprendizaje basado en Proyectos (ABP), el Flipped Classroom y la Gamificación a través de los correspondientes hashtags, tuits y retuits. Los resultados nos permitieron conocer el grado de repercusión y análisis de las relaciones que se establecieron entre los diferentes usuarios de Twitter sobre metodologías activas, así como comprobar cuál de ellas posee una mayor relevancia, y qué o quiénes están detrás de este interés por las metodologías activas. Se concluye que, cuando un usuario adopta un papel protagonista retuiteando tuits de otras cuentas, favorece que los tuits y retuits propios sean más visibles y, a su vez, más retuiteados. Respecto a las estructuras de red que se configuran de cada metodología activa, cabe decir que no siguen un modelo único, aunque todos ellos se ajustan a la clasificación propuesta por Himelboim, Smith, Rainie, Shneiderman y Espina (2017).

Palabras clave: análisis de redes; metodologías activas; tecnología de la información. 


\begin{abstract}
Twitter is a social network where users interact in a common digital space, forming connections that, in themselves, configure, in turn, their own interaction networks based on the exchange of content. This paper has addressed this issue around the impact that active methodologies have on Twitter. For this, we carried out an investigation where the technique of social network analysis was used, both in the analysis of the type of message as well as its graphic representation. The active methodologies analyzed have been project-based learning (PBL), the Flipped Classroom and gamification through the corresponding hashtags, tweets and retweets. The results have allowed us to know the degree of repercussion and analysis of the relationships that have been established between the different Twitter users about active methodologies, as well as to check which of them has greater relevance, and who is behind this interest in the active methodologies. It is concluded that, when a user adopts a leading role by retweeting other account tweets, it favors that the tweets and retweets themselves are more visible and, in turn, more retweeted. Regarding the network structures that are configured for each active methodology, we can say that they do not follow a single model, although all of them conform to the classification proposed by Himelboim, Smith, Rainie, Shneiderman and Espina (2017).
\end{abstract}

Keywords: network analysis; active methodologies; information technology.

La ya afianzada popularidad de las principales plataformas de redes sociales ha propiciado la creación de nuevas redes sociales digitales en las que los individuos interactúan y comparten información y opinión a través de nuevos medios y nuevos códigos (Trottier, 2012). En este sentido, las Tecnologías de la Información y la Comunicación (TIC) han transformado las redes sociales presenciales y han conformado nuevos espacios virtuales donde los usuarios, identificados como nodos, se relacionan a través de la tecnología (Alhabash, 2017). Resulta, pues, interesante ahondar en estos nuevos escenarios, porque, a grosso modo, se ha prestado poca atención a explorar las prácticas de los usuarios en dichas redes sociales y cómo las publicaciones individuales responden a un enfoque interactivo determinado, especialmente, por lo que se refiere a cuestiones directamente vinculadas al contexto educativo, como es el tema de las metodologías activas. Es en este sentido que se ha pretendido llevar a cabo una investigación en torno a dicha temática en la red social Twitter, una de las principales redes que existen actualmente.

\title{
Las redes sociales en el ámbito educativo
}

En el mundo de la educación, el profesorado de las distintas etapas y niveles educativos, desde los maestros y maestras de Infantil al profesorado universitario, tiende a tejer redes de conexión, de establecer relaciones que le permitan, a través de la información y la comunicación que en ellas se establecen, mejorar su aprendizaje 
y establecer vínculos o conexiones con las que compartir recursos, actividades y experiencias (Lytras et al., 2018). Estas redes han visto cómo, con el desarrollo de las Tecnologías de la Información y la Comunicación (TIC) y, más concretamente, con la irrupción de Internet en el mundo educativo, se han establecido amplias redes docentes en las redes sociales más utilizadas (Duart, 2009).

Así, el servicio de microbloging de Twitter ha experimentado, en los últimos años, un aumento considerable, entre sus usuarios, del colectivo docente, generando determinadas audiencias a partir de temas de interés (Litt y Hargittai, 2016). En Twitter el profesorado hace, además de un uso personal, un uso que posibilita el desarrollo profesional (Carpenter y Krutka, 2014; Davis, 2015), siendo éste el uso que adquiere mayor relevancia (Visser, Calvert y Barrett, 2014; Krutka y Carpenter, 2016) y que no es sólo en beneficio propio, sino también llevando a cabo experiencias y prácticas educativas con su alumnado (Grosseck y Holotescu, 2008). Las redes sociales, a su vez, tienen un gran poder de influencia y son generadoras de cambio (Quan-Haasel, Martin y McCay-Peet, 2015). La docencia, la investigación y la cultura en general, están sufriendo cambios que vienen condicionados por el uso que se hace de las redes sociales, pero, sobre todo, se trata de cambios que en el ámbito académico han venido a enriquecer y a potenciar el aprendizaje, la práctica docente y la investigación (Buxarrais, 2016; Rainford, 2016).

Twitter no deja de ser un servicio que ofrece información y comunicación, y es por ello que el profesorado lo utiliza también con este fin. A partir de las cuentas que se siguen, con las que se comparten intereses comunes, un usuario de Twitter puede estar informado acerca de lo que estas cuentas tuitean. Asimismo, cualquier usuario puede lanzar tuits de forma abierta, que serán vistos por todos sus seguidores o, de forma privada, mediante tuits directos a cuentas que se siguen mutuamente (Duart, 2009). En definitiva, los usuarios, al interactuar, en este caso en Twitter, definen determinados canales y flujos de información, los cuales conforman estructuras jerárquicas diferentes a las tradicionales, con base en el número de seguidores, tuits y retuits, comentarios, etc.

Sea de una forma u otra, se establece una comunicación que aporta al docente grandes beneficios, tanto personal como profesionalmente (Choo et al., 2014). Del mismo modo, Twitter es, a su vez, un excelente instrumento de formación permanente (Fernández e Ibáñez, 2011; Abella y Delgado, 2015). En este sentido, una de las temáticas que consideramos interesantes abordar en este contexto es la referida a metodologías activas.

\section{Las metodologías activas a través de Twitter}

Actualmente son muchos los centros educativos, colectivos, administraciones educativas y docentes que, interesados en innovar e introducir cambios para mejorar ciertos aspectos que no funcionan en la educación, se atreven con la implementación de las llamadas metodologías emergentes o metodologías activas (Gros y Noguera, 
2013) que consiguen una mayor implicación del alumnado en su aprendizaje (Benito y Cruz, 2005). Se trata de prácticas educativas, unas más novedosas que otras, donde se da un mayor protagonismo al alumno y se aprende haciendo, indagando e interactuando (Konopka, Adaime y Mosele, 2015), utilizando las TIC de forma coherente (García-Umaña y Tirado-Morueta, 2018).

Las metodologías activas son, pues, todas aquellas actividades, métodos y estrategias que usa el docente para conseguir una mayor participación activa del discente en el proceso de enseñanza-aprendizaje generando en éste un aprendizaje más eficaz. Entre las metodologías activas cabe citar, como ejemplos, el Aprendizaje Basado en Proyectos (ABP), el Aprendizaje Basado en Problemas (ABP), la Gamificación, el Flipped Classroom, el Visual Thinking, el Design Thinking, el Studio Based Learning, el Aprendizaje Colaborativo, etc.

De todas ellas, queremos focalizar nuestra atención en el Aprendizaje Basado en Proyectos, la Gamificación y el Flipped Classroom, tres metodologías activas de las cuales se están abordando numerosos estudios y experiencias educativas (Palma, Lagos y Mora, 2017; Sosa \& Palau, 2018). Además de tener en cuenta el factor referido a una mayor producción científica en su estudio e investigación, también cabe destacar que están adquiriendo en los últimos años un mayor uso por parte de los docentes en los centros educativos de todas las etapas. Buena prueba de esto último es que, con diferencia, son las tres metodologías que mayor número de tuits generan en Twitter, después de haber hecho tal consulta, y, de ahí, nuestra intención de comprobar esta repercusión y protagonismo, así como las relaciones que se establecen entre las cuentas que tuitean sobre ello.

Cuando se habla de redes sociales en el contexto educativo, se hace referencia, normalmente, al uso que de ellas se puede hacer en el aula (Creer, 2018). Así, por ejemplo, se analiza el efecto que tienen sobre el éxito académico del discente (Kirschner y Kirpinski, 2010) o profesional del docente (Túñez y García, 2012), etc. Ahora bien, en este caso, hemos querido centrar nuestra atención sobre las redes sociales y, en concreto, Twitter, puesto que se trata de un instrumento social actual temático en cuanto a la visibilidad y repercusión que ofrece, en este caso, respecto al tema referido a las metodologías activas. En este sentido, debemos recordar que, según el informe de 2018 de Hootsuite (Steel et al., 2017) y We are Social, en julio de 2018 Twitter tenía 336 millones de cuentas en el mundo (https://digitalreport. wearesocial.com/).

Todo ello nos permitirá, además de conocer el grado de repercusión y análisis de las relaciones que se establecen entre los diferentes usuarios de Twitter, poder comprobar cuál de las metodologías activas ha adquirido una mayor relevancia y qué o quiénes están detrás de este interés por dichas metodologías. En este sentido, es interesante analizar si los tuits son originales o bien son retuits, "lo que demostraría una estrategia de creación propia en redes sociales, frente a una en la que simplemente se dedique a compartir contenidos publicados por otros" (Fernández-Gómez y Martín-Quevedo, 2018, p. 1294). 
Por todo esto, las preguntas de investigación que nos planteamos son:

- ¿Qué grado de repercusión, medido en cantidad de tuits y relaciones entre cuentas de Twitter se generan alrededor de las metodologías activas ABP, la Gamificación y Flipped Classroom?

- ¿Qué red de relaciones se ha creado en Twitter en base a la temática de las metodologías activas?

- ¿Cuál es el perfil de los principales usuarios de Twitter que abordan el tema de las metodologías activas?

\section{METODOLOGIA}

\section{Contexto de la investigación}

El objetivo de la presente investigación estriba en conocer el grado de repercusión que tienen actualmente en Twitter las siguientes metodologías activas: el ABP, el Flipped Classroom y la Gamificación. Para conocer este grado de repercusión, así como las relaciones que se establecen entre los diferentes usuarios participantes en esta difusión, utilizaremos la técnica del análisis de redes sociales (ARS) (Leonardi y Renée, 2018). Esta técnica ha despertado, en los últimos años, un creciente interés en el ámbito de estudio de las Ciencias Sociales, pues, gracias a ella, se consiguen establecer relaciones entre los diferentes usuarios protagonistas, así como conocer su comportamiento social (Kümpel, Karnowski y Keyling, 2015). Con ello, se supera el considerar únicamente los atributos individuales que se conseguían con las investigaciones tradicionales. Aun así, es difícil recoger datos de Twitter, especialmente para estudios en el ámbito de la psicopedagogía (Murphy, 2017).

Para ello, hemos querido basarnos en la teoría de redes "mediante la cual es posible comprender y modelar sistemas complejos [los cuales pueden ser visualizados a través de] diferentes tipos de grafos" (Romero-Frías y Arroyo-Machado, 2018, p. 1183) donde los individuos constituyen los nodos y las relaciones entre ellos, las aristas. En este caso, se analizó la red de información con base en el flujo de interacciones producidas por medio de los tuits y retuits, es decir, se utilizaron datos cuantitativos de la referida técnica ARS, "que posibilita entender la estructura y los componentes que forman los mensajes” (Alonso-Muñoz y Casero-Ripollés, 2018, p. 1195). Por otro lado, se analizaron las relaciones sociales entre los usuarios a partir de las conexiones de seguimiento establecidas (Himelboim, Smith, Rainie, Shneiderman y Espina, 2017). 


\section{Instrumento de evaluación y procedimiento}

Para la extracción de datos de Twitter, se utilizó la aplicación T-hoarder_kit. Con ella se extrajeron datos de los tuits y los retuits, así como de las cuentas que los realizaron. Se utilizaron los hashtags reconocidos para las metodologías activas a estudiar (ver tabla 1): ABP, Flipped Classroom y Gamificación. Cabe decir, sin embargo, que, en un principio, se trató de estudiar el comportamiento y repercusión en Twitter de un abanico amplio de metodologías activas, como visual thinking, trabajo colaborativo y cooperativo, etc., pero se desestimaron, dada la poca relevancia de los datos que se obtuvieron a partir de los hashtags correspondientes.

Tabla 1. Hashtags utilizados para cada metodología

\begin{tabular}{|l|l|}
\hline \multicolumn{1}{|c|}{ Metodología } & \multicolumn{1}{c|}{ Hashtags } \\
\hline Aprendizaje Basado en Proyectos & $\begin{array}{l}\text { \#ABP, \#ABP_es, \#PBL_es, } \\
\text { \#trabajoporproyectos, } \\
\text { \#aprendizajebasadoenproyectos }\end{array}$ \\
\hline Flipped Classroom & $\begin{array}{l}\text { \#flippedclassroom, \#flippedlearning, } \\
\text { \#flipped }\end{array}$ \\
\hline Gamificación & \#gamificación, \#gamificacion \\
\hline
\end{tabular}

Se realizaron tres búsquedas y se extrajeron datos, descartando aquellos correspondientes a cuentas, tuits y retuits ajenos a las metodologías objeto de investigación. La purga de datos fue bastante cuidadosa en el caso del Aprendizaje basado en Proyectos, pues el hashtag \#ABP responde a diversas acepciones, tanto en el contexto educativo (p. e., Aprendizaje basado en Problemas) como otros contextos (p. e.: Asociación de Bodyboarders de Panamá). Cada búsqueda incluía todos los tuits y retuits que, con dichos hashtags, se realizó entre el 28 de noviembre y el 8 de diciembre de 2018.

A partir de los retuits realizados con los hashtags definidos (en este caso, los que aparecen en la tabla 1), se obtienen las relaciones entre cuentas de Twitter. Estas nos permiten, a través del programa Gephi, sobre tratamiento de datos relacionales, obtener los diferentes grafos para cada tema, en este caso, para cada metodología activa. Así mismo, la cantidad de tuits y retuits realizados por cada una de las cuentas implicadas, se traduce gráficamente en nodos con un mayor o menor tamaño en función del número de éstos.

Se obtiene, así, una abstracción que representa una red, donde los nodos o vértices, que son las diversas cuentas de Twitter, se unen mediante aristas o enlaces que representan las relaciones que se establecen entre ellas. De esta manera, los grafos nos permiten identificar las características estructurales de las redes para cada metodología y se puede comprobar la repercusión que tiene cada una de ellas en Twitter. Este análisis se realizó a partir de un enfoque global, teniendo en cuenta 
toda la red, tanto el conjunto de nodos como de aristas. Asimismo, las relaciones establecidas con los retuits obedecen a todos los casos en los que se produce un retuit, independientemente de si incluye o no algún comentario añadido.

Una vez realizado el análisis indicado, se procede a configurar el grafo resultante, el cual debe ser analizado. Para ello, nos basaremos en la clasificación de Himelboim et al. (2017), según los cuales dichos grafos pueden responder a diversos tipos de estructuras de redes sociales de Twitter: a) Hub-and-Spoke: Broadcast and Support. Es una red caracterizada por un alto grado de centralización, con estructuras en forma de estrella, donde se da una concentración de poder en manos de muy pocos (hacia adentro y hacia afuera); b) Fragmented: Brand Clusters. Este modelo tiene un gran número de personas que no tienen ninguna conexión (aislamientos); c) Divided: Polarized Clusters. Son redes que se caracterizan por una alta densidad de gráficos (polarización). Se forman a partir de pocos grupos, pero grandes y densos, con poca conexión entre ellos.; d) Unified: In-Group. Son redes caracterizadas por una estructura de grupo, donde los usuarios están altamente interconectados y esencialmente se superponen entre sí; e) Clustered: Community Clusters. Son redes con baja densidad de gráficos y baja porción de aislamientos, a modo de grupos comunitarios. Son similares a las del modelo fragmented Brand, pero la cantidad de aislamientos es inferior.

\section{RESULTADOS}

Una vez tratados los datos recogidos a partir de la aplicación T-hoarder_kit y establecidas las relaciones dirigidas (retuits) entre las diferentes cuentas de Twitter o usuarios implicados, podemos observar en la tabla 2 los datos referidos a las cuentas de Twitter con mayor número de retuits referidos a la metodología ABP.

Tabla 2. Tuits, retuits, y detalles de las cuentas implicadas en ABP

\begin{tabular}{|l|c|c|c|c|c|c|}
\hline Cuenta/Usuario & $\begin{array}{c}\text { Tuits + RTs } \\
\text { realizados }\end{array}$ & $\begin{array}{c}\text { total de } \\
\text { RTs }\end{array}$ & $\begin{array}{c}\text { RTs } \\
\text { recibidos }\end{array}$ & $\begin{array}{c}\text { RTs } \\
\text { realizados }\end{array}$ & seguidores & seguidos \\
\hline @educacion3_o & 9 & 127 & 127 & 0 & 147875 & 10818 \\
\hline @cedec_intef & 12 & 45 & 45 & 0 & 54142 & 26870 \\
\hline @escolahoritzo & 5 & 44 & 44 & 0 & 764 & 374 \\
\hline @jdomenechca & 1 & 28 & 28 & 0 & 2657 & 97 \\
\hline @proyectoedia & 20 & 20 & 0 & 20 & 1028 & 999 \\
\hline @javieducacio & 1 & 19 & 18 & 1 & 1643 & 362 \\
\hline @cfie_cr & 5 & 19 & 17 & 2 & 1730 & 1381 \\
\hline @marmarmur & 3 & 18 & 17 & 1 & 3540 & 1345 \\
\hline @colegioamoros & 8 & 17 & 17 & 0 & 2842 & 280 \\
\hline @noupatufet & 6 & 14 & 14 & 0 & 1201 & 586 \\
\hline
\end{tabular}




\begin{tabular}{|l|c|c|c|c|c|c|}
\hline Cuenta/Usuario & $\begin{array}{c}\text { Tuits + RTs } \\
\text { realizados }\end{array}$ & $\begin{array}{c}\text { total de } \\
\text { RTs }\end{array}$ & $\begin{array}{c}\text { RTs } \\
\text { recibidos }\end{array}$ & $\begin{array}{c}\text { RTs } \\
\text { realizados }\end{array}$ & seguidores & seguidos \\
\hline Cuenta/Usuario & $\begin{array}{c}\text { Tuits + RTs } \\
\text { realizados }\end{array}$ & $\begin{array}{c}\text { total de } \\
\text { RTs }\end{array}$ & $\begin{array}{c}\text { RTs } \\
\text { recibidos }\end{array}$ & $\begin{array}{c}\text { RTs } \\
\text { realizados }\end{array}$ & seguidores & seguidos \\
\hline @jarevalo_fp & 2 & 14 & 14 & 0 & 11523 & 6677 \\
\hline @eraser & 6 & 13 & 13 & 0 & 24350 & 12890 \\
\hline @cfiecr_tic & 6 & 13 & 10 & 3 & 299 & 333 \\
\hline @danimartic & 1 & 12 & 12 & 0 & 3274 & 2549 \\
\hline @isahenares & 1 & 10 & 10 & 0 & 194 & 293 \\
\hline
\end{tabular}

Si nos fijamos en las quince primeras cuentas con mayor repercusión, en su mayoría se trata de cuentas a las que se retuitean sus tuits, sin que éstas retuiteen los tuits de otras cuentas relacionadas con la metodología ABP. Numéricamente, los valores van desde los 127 hasta los 10 retuits. La primera cuenta, “@educacion3_o”, corresponde al perfil de una revista de tecnología educativa que habitualmente lanza tuits referidos a contenidos que va publicando en sus ediciones, tanto en su versión digital como impresa. Esta cuenta recibe un total de 127 retuits sobre los 9 tuits originales que lanza sobre ABP pero, teniendo en cuenta el gran número de seguidores, en total 147.875, su efectividad es del 0,77\%. En términos muy similares, estaríamos hablando de la cuenta del Centro Nacional de Desarrollo Curricular en Sistemas no Propietarios, “@cedec_intef”, que alcanza una efectividad del o,99\% con 12 tuits. Sin embargo, la cuenta referida a un usuario individual, “@jdomenechca”, con un solo tuit logra 28 retuits, lo cual le confiere una efectividad del 1,05\%. Destaca también la cuenta de “@proyectoedia”, que únicamente retuitea tuits de otros usuarios.

En el grafo de la figura 1 podemos, además, comprobar cómo la cuenta de “@ educacion3_o" está relacionada con las otras cuentas, en su mayoría, de una forma directa y, en otros muy pocos casos, de forma indirecta. Destaca nuevamente la cuenta “@jdomenechca”, ya que consigue tejer unas relaciones directas con las principales cuentas, mientras que cuentas como “@escolahoritzo”, “@red_escuelas” o "@javieducacio" quedan más aisladas y con muy poca relación con las cuentas principales. Aun con todo ello, las relaciones que cada una de las cuentas principales establecen, lo hacen con otras cuentas y existe muy poca relación entre ellas. De ahí que las aristas (relaciones) que las unen queden tan solitarias y que las que establecen con las otras cuentas queden tan concéntricas alrededor del nodo (cuenta o usuario). 
Figura 1. Grafo con las relaciones sobre ABP

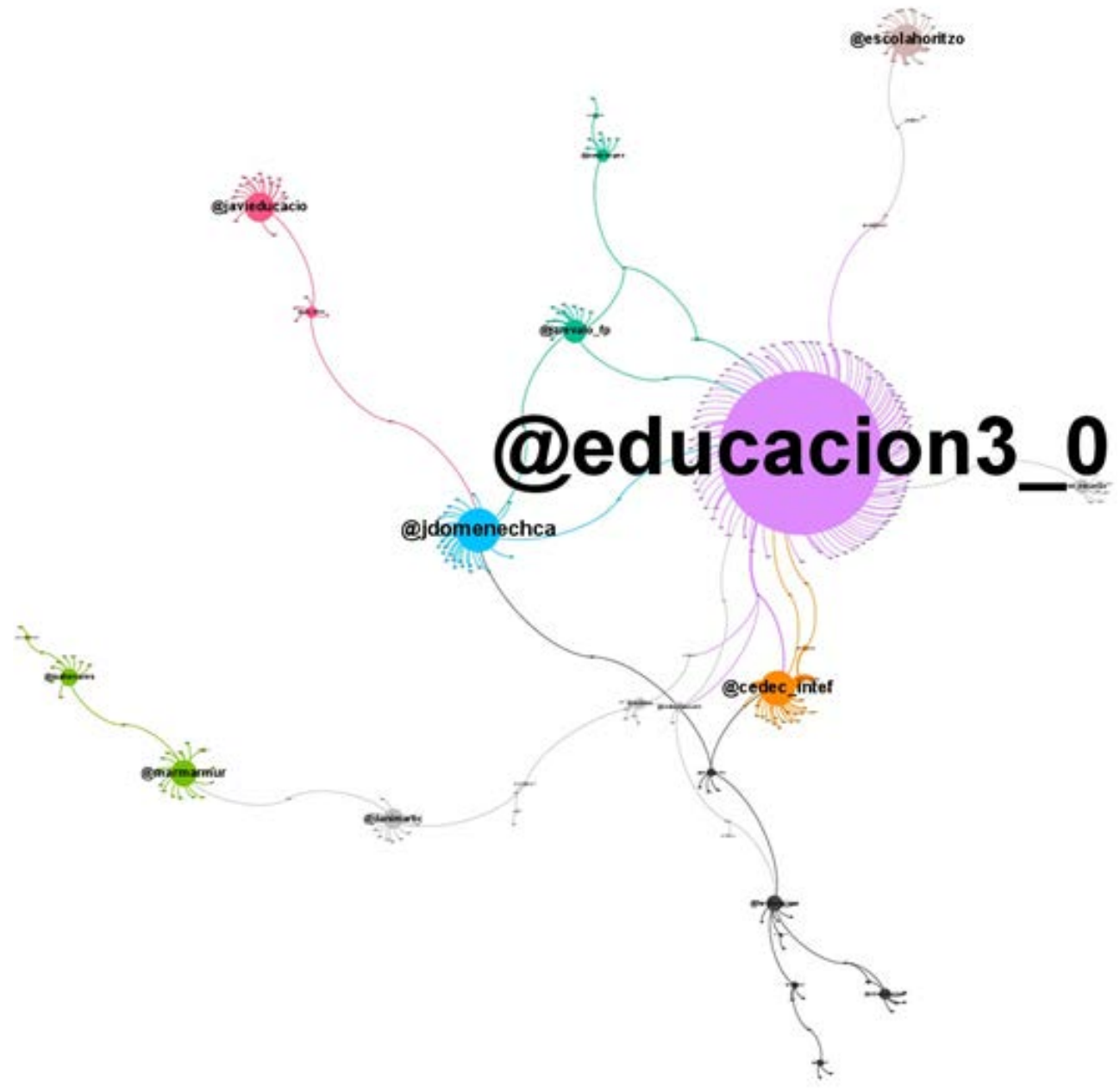

Con respecto a la metodología Flipped Classroom, se puede observar en la tabla 3 las cuentas de Twitter con mayor número de retuits respecto a los hashtags analizados. Si contemplamos estas quince primeras cuentas que establecen relaciones, vemos que la repercusión del Flipped Classroom respecto al ABP es mucho menor, pues genera un número de relaciones inferior. El máximo de dichas relaciones en estas primeras quince cuentas se fija en 36 y el mínimo en 5 . Se han eliminado aquellas cuentas que, a pesar de llevar el hashtag o hashtags de esta metodología, se referían a cuentas 
que comunicaban en inglés y tenían un ámbito de relación y repercusión fuera de España. Ahora bien, aunque se hubieran contemplado, no hubiese cambiado apenas la repercusión o el grado de influencia referida al Flipped Classroom en Twitter, ya que ocupaban puestos en la lista de cuentas con mayor número de relaciones por debajo de la quinta posición y se encontraban alternas con las otras cuentas que sí han permanecido en este ranking.

Tabla 3. Tuits, retuits, y detalles de las cuentas implicadas en Flipped Classroom

\begin{tabular}{|l|c|c|c|c|c|c|}
\hline Cuenta/Usuario & $\begin{array}{c}\text { Tuits + RTs } \\
\text { realizados }\end{array}$ & $\begin{array}{c}\text { total de } \\
\text { RTs }\end{array}$ & $\begin{array}{c}\text { RTs } \\
\text { recibidos }\end{array}$ & $\begin{array}{c}\text { RTs } \\
\text { realizados }\end{array}$ & seguidores & seguidos \\
\hline @simplificamates & 3 & 36 & 35 & 1 & 1056 & 214 \\
\hline @sanmartinjuan & 6 & 35 & 32 & 3 & 1535 & 1258 \\
\hline @jesus_aceitunod & 2 & 15 & 14 & 1 & 1460 & 892 \\
\hline @netlearnig20 & 5 & 11 & 10 & 1 & 6962 & 1290 \\
\hline @isabelgarciasvp & 5 & 11 & 7 & 4 & 2069 & 674 \\
\hline @antonio_guirao & 4 & 10 & 10 & 0 & 10296 & 5659 \\
\hline @migueldoceo & 1 & 9 & 9 & 0 & 236 & 244 \\
\hline @ramon3434 & 26 & 8 & 8 & 0 & 16894 & 14664 \\
\hline @classroomcrafts & 4 & 8 & 4 & 4 & 2409 & 1102 \\
\hline @ordifilosofo & 1 & 7 & 7 & 0 & 1229 & 334 \\
\hline @mathigaele & 1 & 7 & 7 & 0 & 932 & 1554 \\
\hline @elpatiodeoscar & 2 & 7 & 6 & 1 & 409 & 370 \\
\hline @tic_fecc & 4 & 6 & 6 & 0 & 1627 & 438 \\
\hline @tonibernabeu123 & 1 & 5 & 5 & 0 & 3482 & 771 \\
\hline @juanfisicahr & 5 & 5 & 0 & 5 & 4265 & 900 \\
\hline
\end{tabular}

En el grafo de la figura 2, se puede observar las relaciones (aristas) que se establecen entre las diferentes cuentas (nodos) y vemos que, si bien las dos primeras cuentas con mayor número de relaciones (@simplificamates y @sanmartinjuan) aparecen bien posicionadas, no ocurre lo mismo con la tercera y cuarta (@jesus_ aceitunod y @netlearning20), que no mantienen relaciones con las dos primeras o las restantes cuentas principales. Por ello, su presencia en el grafo es apenas testimonial. Sin embargo, precisamente estas conexiones o relaciones hacen que, cuentas que no aparecen entre las quince primeras en número de retuits, sí lo hagan en el grafo, debido a la relevancia de sus conexiones (es el caso de @dchicapardo, @ maths_revolutio o @colquiga). 
Figura 2. Grafo con las relaciones sobre Flipped Classroom

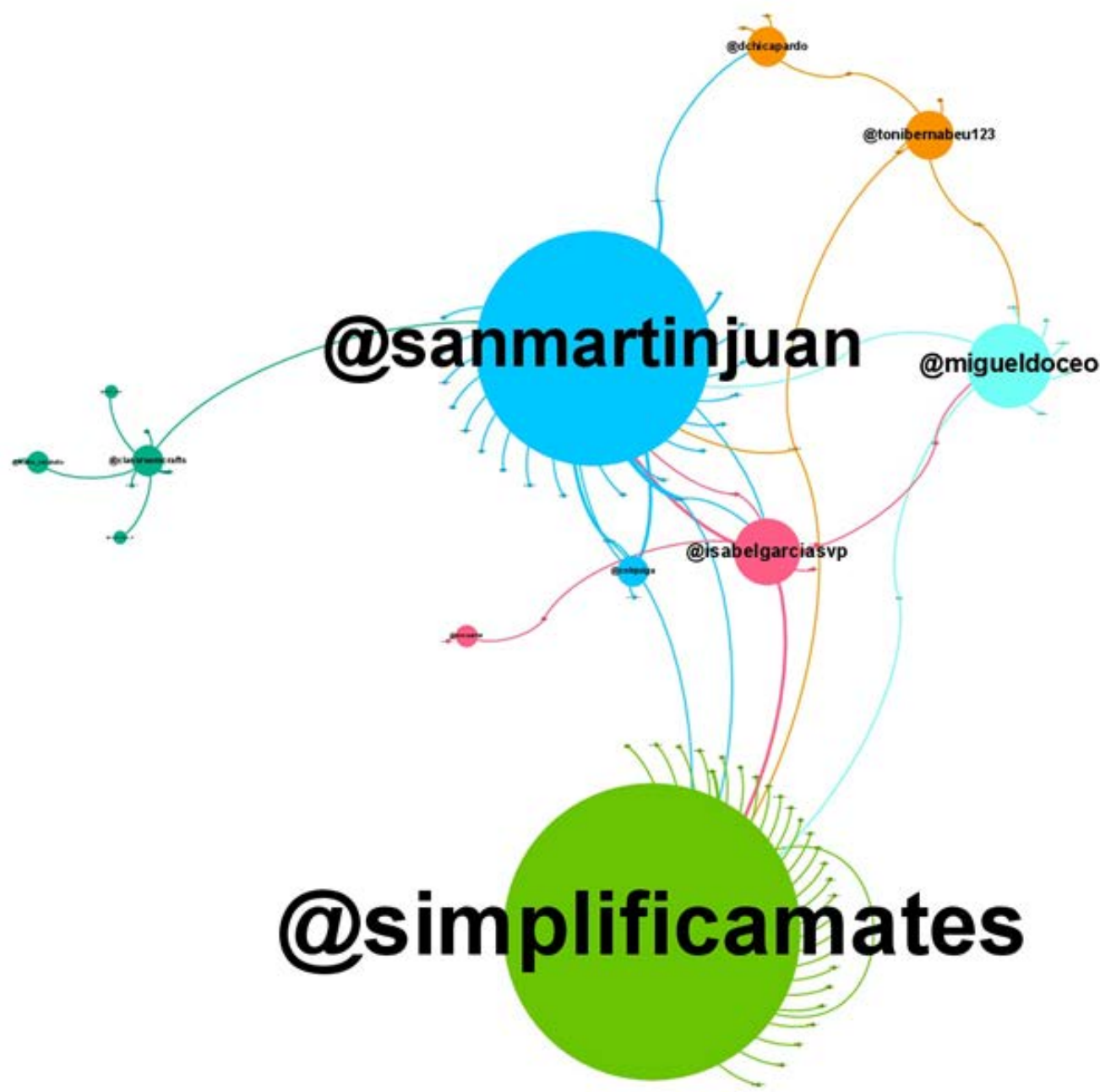

En cuanto a la última metodología activa que se ha considerado, la Gamificación, cabe decir que, tal y como se puede apreciar en la tabla 4, las cuatro primeras cuentas se encuentran por encima de los 100 retuits, siendo el máximo de 790 y el mínimo de 22.

Destaca la primera cuenta (@ classroomcrafts, con 2.405 seguidores y que sigue a 1.102 cuentas), la cual tiene 790 retuits, 275 de los cuales son recibidos y 515 realizados. Respecto a su eficacia, cabe decir que, a partir de 2 tuits y 515 retuits comentados, 
consigue un total de 790 retuits. Respecto a la segunda cuenta (@ludigencia), que únicamente lanza tuits y no retuitea, y la tercera cuenta (jorgetovilla_), que sólo retuitea mensajes de otras cuentas, pasan desapercibidas en cuanto a relaciones con las principales cuentas que tuitean sobre el tema de Gamificación.

En un tercer plano, pero no por ello menos importante, se encuentran cuentas como @educacion3_o que, gracias a su elevado número de seguidores, consigue con 8 tuits que estos sean retuiteados 126 veces. Otras cuentas que le siguen, como @ fernando_marti7, @mrcuarter y @maria_glvez, con un menor número de seguidores y con solo algunos tuits ( 3,1 y 3 respectivamente), consiguen un elevado número de retuits, lo cual les hace sumar importancia.

Tabla 4. Tuits, retuits, y detalles de las cuentas implicadas en Gamificación

\begin{tabular}{|l|c|c|c|c|c|c|}
\hline Cuenta/Usuario & $\begin{array}{c}\text { Tuits + RTs } \\
\text { realizados }\end{array}$ & $\begin{array}{c}\text { total de } \\
\text { RTs }\end{array}$ & $\begin{array}{c}\text { RTs } \\
\text { recibidos }\end{array}$ & $\begin{array}{c}\text { RTs } \\
\text { realizados }\end{array}$ & seguidores & seguidos \\
\hline @classroomcrafts & 517 & 790 & 275 & 515 & 2405 & 1102 \\
\hline @ludigencia & 455 & 372 & 372 & 0 & 2810 & 2797 \\
\hline @jorgetovilla_ & 208 & 208 & 0 & 208 & 20774 & 447 \\
\hline @educacion3_o & 8 & 126 & 126 & 0 & 147923 & 10819 \\
\hline @fernando_marti7 & 3 & 95 & 94 & 1 & 1288 & 629 \\
\hline @mrcuarter & 1 & 55 & 55 & 0 & 2531 & 738 \\
\hline @maria_glvez & 3 & 48 & 48 & 0 & 4289 & 882 \\
\hline @genially_es & 10 & 42 & 38 & 4 & 12154 & 5530 \\
\hline @eraser & 13 & 34 & 34 & 0 & 24363 & 12909 \\
\hline @ramon3434 & 40 & 30 & 30 & 0 & 16896 & 14663 \\
\hline @azaharatic & 3 & 30 & 28 & 2 & 6010 & 956 \\
\hline @elmformacion & 15 & 29 & 22 & 7 & 4421 & 4997 \\
\hline @cookiebox_sl & 9 & 28 & 28 & 0 & 1488 & 101 \\
\hline @manuparadas & 2 & 23 & 22 & 1 & 6501 & 973 \\
\hline @toolkitseu & 10 & 22 & 22 & 0 & 553 & 482 \\
\hline
\end{tabular}

De manera gráfica, se puede observar en la figura 3 que la cuenta @ classroomcrafts presenta una sólida relación con la gran mayoría de cuentas activas respecto a los hashtags de esta metodología. Sus ramificaciones conectan con las otras cuentas, generando una estructura desarrollada y no aislada respecto al grafo global de relaciones. En este caso, vemos que la cuenta @educacion3_o, a pesar de tener un elevado número de ramificaciones, queda bastante desconectada de las otras cuentas y aparece en un lateral del grafo. 
Figura 3. Grafo con las relaciones sobre Gamificación

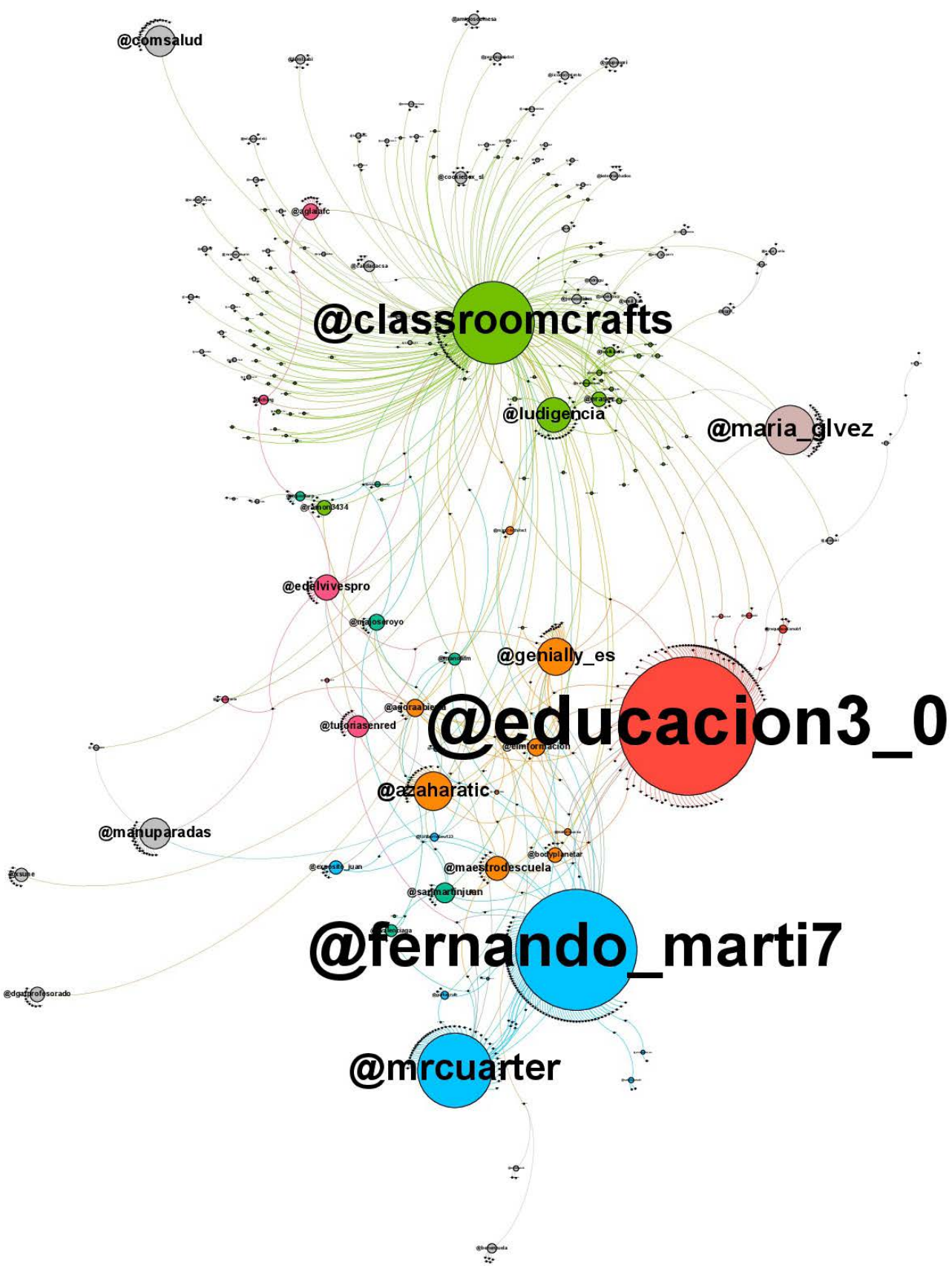


En el caso de cuentas como las de @fernando_marti7, @mrcuarter y @maria_ glvez, aunque poseen pocos seguidores y han realizado pocos tuits, tienen sólidas relaciones, tal y como se observa en el grafo. Ello es fruto de la eficacia de éstas, ya que con pocos tuits y pocos seguidores, consiguen un elevado número de retuits. En el extremo opuesto, encontramos otras cuentas que, bien no aparecen en el grafo, mientras sí lo hacían en la tabla 4 referida a las 15 cuentas con mayor número de retuits (@genially_es, @eraser o @ramon3434), bien aparecen en el grafo, pero no tienen un número considerable de retuits que les permita aparecer en la tabla 4 (@ comsalud, @edelvivespro o @maestrodeescuela).

\section{DISCUSIÓN Y CONCLUSIONES}

El análisis que hemos realizado muestra una visión empírica de las interacciones en la red social Twitter en torno al tema de las metodologías activas. En este sentido, Twitter ofrece una herramienta de comunicación que amplifica el alcance de cualquier información y, por ello, se ha considerado adecuado/conveniente analizar esta cuestión en concreto. Hemos realizado, de esta manera, un estudio interaccional en Twitter focalizado en las metodologías activas. Para tal fin, se ha ilustrado nuestro análisis a partir de tres de ellas -ABP, Flipped Classroom y Gamificacióndesarrollando la categorización de los tuits a partir de hashtags y construyendo un gráfico esquemático para estudiar la interacción de los usuarios, en concordancia con estudios anteriores (Romero-Frías y Arroyo-Machado, 2018).

Respecto a la pregunta de investigación referida a cuál es el perfil de los principales usuarios de Twitter que abordan el tema de las metodologías activas, cabe decir que, con respecto a la Gamificación, destaca la cuenta (@ classroomcrafts) con 790 retuits, 275 de los cuales son recibidos y 515 realizados. Se observa con ello que tener un papel protagonista retuiteando tuits de otras cuentas, favorece que los tuits y retuits propios sean más visibles y a su vez retuiteados. Estamos hablando de una cuenta que no tiene cifras muy elevadas de seguidores y seguidos, ya que sólo tiene 2.405 seguidores y sigue a 1.102 cuentas, pero desempeñar un papel muy activo en Twitter hace que aumente considerablemente sus conexiones/relaciones.

En el caso de cuentas como las de @fernando_marti7, @mrcuarter y @maria_ glvez, aunque poseen pocos seguidores y han realizado pocos tuits, tienen sólidas relaciones. Ello es fruto de la eficacia de éstas ya que, con pocos tuits y pocos seguidores, consiguen un elevado número de retuits. Otra muestra de la calidad de las cuentas que siguen y son seguidas por estos nodos. Respecto al Flipped Classroom, se observa que el tráfico de hashtags se concentra en cuentas individuales, personales y de docentes, y no de revistas, centros educativos u organismos y centros de la administración educativa, como sí ocurría en la metodología ABP. Además, cabe decir que es, de las tres metodologías activas analizadas, la que mayor repercusión posee debido al número de relaciones establecidas, destacando notablemente/ significativamente con respecto al ABP y a la Gamificación. 
En cuanto a la pregunta de investigación referida a qué red de relaciones se ha creado en Twitter en base a la temática de las metodologías activas, cabe indicar que, a partir de la clasificación de Himelboim et al. (2017), el grafo resultante del ABP se acerca al modelo denominado Broadcast, ya que la cuenta @educacion3_o y todas las conexiones que parten de ella funcionan según dicho modelo. Ahora bien, según el resto -0 , incluso, el conjunto en general-, estaríamos hablando del modelo Brand Clusters, con nodos aislados y poco conectados entre ellos, como @ jdomenechca, @cedec_intef o @escolahoritzo. En lo concerniente con el Flipped Classroom, no podemos definirlo claramente según el modelo Polarized y, más bien, se podría clasificar siguiendo el modelo Brand Clusters. Por último, cabe decir que la Gamificación se aproxima más al modelo Community Clusters y no tanto al Brand Clusters, ya que hay bastantes interconexiones entre los diferentes nodos.

Finalmente, cabe decir que el estudio tiene sus propias limitaciones: algunas de ellas derivadas de la presencia desigual en Twitter de los diversos usuarios analizados, y otras de la acotación de hashtags según el idioma. En futuras investigaciones, se podría investigar en torno a los hashtags en otros idiomas, realizar estudios longitudinales y también utilizando, además, otros métodos de análisis de las redes generadas en Twitter. Por otro lado, sabemos que no toda la comunidad docente está presente -o activa- en las redes sociales y que hay muchos docentes que innovan, realizan buenas prácticas y utilizan metodologías activas en su aula sin que nadie ajeno a su entorno más cercano sepa de ello. Este artículo no se ha focalizado en esta población, sino en los usuarios de Twitter vinculados activamente en las denominadas metodologías activas, de lo cual se ha realizado el estudio.

\section{AGRADECIMIENTO}

El presente trabajo se enmarca en el seno del Grupo de Investigación "EDUTICADEI" (Ref.: Vigrob-039), del Programa de Redes de investigación en docencia universitaria del ICE y del proyecto Instituto Superior de Investigación Cooperativa IVITRA(Ref.: ISIC/2012/022; http://www.ivitra.ua.es), todos ellos dela Universidad de Alicante (UA); y del grupo de investigación EduInnovagogía ${ }^{\circledR}$ (HUM-971), grupo reconocido por el Plan Andaluz de Investigación, Desarrollo e Innovación y la Oficina de Transferencia de Resultados de Investigación de la Universidad Pablo de Olavide.

\section{REFERENCIAS}

Abella, V., y Delgado, V. (2015). Aprender a usar twitter y usar twitter para aprender. Profesorado. Revista de currículum y formación del profesorado, 19(1), 364378.
Abidin, C. (2016). Aren't These Just Young, Rich Women Doing Vain Things Online?: Influencer Selfies as Subversive Frivolity. Social Media + Society, 1-17. doi: 10.1177/2056305116641342. 
Alhabash, S., y Ma, M. (2017). A Tale of Four Platforms: Motivations and Uses of Facebook, Twitter, Instagram and Snapchat Among College Students? Social Media + Society, 1-13. doi: 10.1177/2056305117691544.

Alonso-Muñoz, L., y Casero-Ripollés, A. (2018). Communication of European populist leaders on Twitter: Agenda setting and the "more is less" effect. $E l$ profesional de la información, 27(6), 1193-1202. Recuperado de http://www. elprofesionaldelainformacion.com/ contenidos/2018/nov/03.pdf

Benito, A., y Cruz, A. (2005). Nuevas claves para la docencia universitaria. Madrid: Narcea.

Buxarrais, M. R. (2016). Redes sociales y educación. Education in the Knowledge Society, 17(2), 15-20.

Carpenter, J. P., y Krutka, D. G. (2014). How and Why Educators Use Twitter: A Survey of the Field. Journal of Research on Technology in Education, 46(4), 414434. doi: https://doi.org/10.1080/153915 23.2014.925701

Choo, E. K., Ranney, M. L., Chan, T. M., Trueger, N. S., Walsh, A. E., Tegtmeyer, K., ... Carroll, C. L. (2014). Twitter as a tool form communication and knowledge exchange in academic medicine: A guide for skeptics and novices. Medical Teacher, 37(5), 411-416. doi: https://doi. org/10.3109/0142159X.2014.993371

Creer, A. (2018). Introducing Everyday 'Digital Literacy Practices' into the Classroom: an Analysis of Multi-layered Media, Modes and their Affordances. Journal of New Approaches in Educational Research, 7(2), 131-139. doi: 10.7821/naer.2018.7.265.

Davis, K. (2015). Teachers' perceptions of Twitter for professional development. Disability and rehabilitation, 37(17), 1551-1558.

Duart, J. (2009). Internet, redes sociales y educación. RUSC. Universities and
Knowledge Society Journal, 6(1). Recuperado de https://www.redalyc.org/ articulo.oa?id $=78011179001$

Fernández, J. J., e Ibáñez Luque, L. (2011). Pedagogía crítica y Web 2.0: formación del profesorado para transformar el aula. Revista electrónica interuniversitaria de formación del profesorado, 37(14-2), 8195.

Fernández-Gómez, E., y Martín-Quevedo, J. (2018). La estrategia de engagement de Netflix España en Twitter. El profesional de la información, 27(6), 1292-1302. Recuperado de http://www. elprofesionaldelainformacion.com/ contenidos/2018/nov/12.pdf

García-Umaña, A., y Tirado-Morueta, R. (2018). Digital Media Behavior of School Students: Abusive Use of the Internet. Journal of New Approaches in Educational Research, 7(2), 140-147. doi: 10.7821/naer.2018.7.284.

Gros, B., y Noguera, I. (2013). Mirando el futuro: Evolución de las tendencias tecnopedagógicas en educación superior. Campus Virtuales, 2(2), 130-140.

Grosseck, G., y Holotescu, C. (2008). Can we use Twitter for educational activities? In $4^{\text {th }}$ International scientific conference, eLearnig and software for education, Bucharest, Romania.

Himelboim, I., Smith, M. A., Rainie, L., Shneiderman, B., y Espina, C. (2017). Classifying Twitter Topic-Networks Using Social Network Analysis. Social Media + Society, 1-13. doi: 10.1177/2056305117691545.

Housley, W., Webb, H., Williams, M., Procter, R., Edwards, A., Jirotka, M., y Williams, M. (2018). Interaction and Transformation on Social Media: The Case of Twitter Campaigns. Social Media + Society, 1-12. doi: https://doi. org $/ 10.1177 / 2056305117750721$

Kirschner, P., y Kirpinski, A. (2010). Facebook and academic performance. 
Computer in Human Behaviors, 26, 12371245 .

Konopka, C. L., Adaime, M. B., y Mosele, P. H. (2015). Active teaching and learning methodologies: some considerations. Creative Education, 6(14), 1536-1545.

Krutka, D. G., y Carpenter, J. P. (2016). Participatory Learning Through Social Media: How and Why Social Studies Educators Use Twitter. Contemporary Issues in Technology and Teacher Education, 16(1), 38-59.

Kümpel, A. S., Karnowski, V., y Keyling, T. (2015). News Sharing in Social Media: A Review of Current Research on News Sharing Users, Content and Networks. Social Media + Society, 1-14. doi: 10.1177/2056305115610141.

Leonardi, V. S., y Renée Elías, S. (2018). Redes sociales en Internet en el marco del turismo cultural: los museos en Bahía Blanca (Argentina). REDES. Revista hispana para el análisis de las redes sociales, 29(1), 92-110. doi: https://doi. org/10.5565/rev/redes.732

Lytras, M. D., Visvizi, A., Daniela, L., Sarirete, A., y De Pablos, P. (2018). Social Networks Research for Sustainable Smart. Sustainability, 1O(9), 2974. doi: 10.3390/ su10092974.

Litt, E., y Hargittai, E. (2016). The Imagined Audience on Social Network Sites. Social Media +Society, 1-12. doi: 10.1177/2056305116633482.

Murphy, S. C. (2017). A Hands-On Guide to Conducting Psychological Research on Twitter. Social Psychological and Personality Science, 8(4), 396-412. doi: 10.1177/1948550617697178.

Palma Luengo, M., Lagos San Martín, N., y Mora Donoso, M. (2017). Metodologías activas para la formación universitaria. Chile: Universidad del Bío-Bío. Recuperado de http://bit.ly/2BA7uTi

Quan-Haasel, A, Martin, K., y McCayPeet, L. (2015). Networks of digital humanities scholars: The informational and social uses and gratifications of Twitter. Big Data \& Society, 1-12. doi: 10.1177/2053951715589417.

Rainford, J. (2016). Becoming a doctoral researcher in a digital world: Reflections on the role of Twitter for reflexivity and the internal conversation. E-Learning and Digital Media, 13(1-2). 99-105. doi: https://doi. org/10.1177/2042753016672380

Romero-Frías, E., y Arroyo-Machado, W. (2018). Policy labs in Europe: Political innovation, structure and content analysis on Twitter. El profesional de la información, 27(6), 11811192. Recuperado de http://www. elprofesionaldelainformacion.com/ contenidos/2018/nov/02.pdf

Romero-Frías, E., y Arroyo-Machado, W. (2018). Policy labs in Europe: political innovation, structure and content analysis on Twitter. El profesional de la información, 27(6), 1181-1192. doi: https://doi.org/10.3145/epi.2018.nov.02

Sosa Díaz, M. J., y Palau Martín, R. F. (2018). Flipped Classroom para adquirir la competencia digital docente: una experiencia didáctica en la Educación Superior. Píxel-Bit. Revista de Medios y Educación, 52, 37-54. doi: http://dx.doi. org/10.12795/pixelbit.2018.i52.03

Steel, E., Berube, Y., Boner, J., Britton, K., y Coatta, T. (2017). Hootsuite: In Pursuit of Reactive Systems. Communications of the $A C M, 60(11), 36-43$. doi: 10.1145/3106627.

Trottier, D. (2012). Social media as surveillance. Farnham, UK: Ashgate.

Túñez, M., y García, J. (2012). Las redes sociales como entorno docente: análisis del uso de Facebook en la docencia universitaria. Pixel Bit, Revista de Medios y Educación, 41, 77-92.

Visser, R. D., Calvert, L., y Barrett, D. E. (2014). \#TwitterforTeachers: The Implications of Twitter as a Self-Directed Professional Development Tool for K-12 Teachers. Journal of Research on 
Technology in Education, 46(4), 396-413. $\quad$ doi: https://doi.org/10.1080/15391523.2 $\underline{014.925694}$

\section{PERFIL ACADÉMICO Y PROFESIONAL DE LOS AUTORES}

Rosabel Roig Vila. Doctora en Pedagogía y Profesora Titular de la Universidad de Alicante del Área de Didáctica y Organización Escolar. Ha sido Decana de la Facultad de Educación y actualmente es la Directora del Instituto de Ciencias de la Educación en esta universidad. Es Directora de la Journal of New Approaches in Educational Research y Coordinadora del grupo de investigación EDUTIC-ADEI (Educación y TIC-Atención a la Diversidad. Escuela Inclusiva). Su investigación se centra en la articulación de las TIC en educación. Ver más información en http:// www.rosabelroigvila.es

E-mail: rosabel.roig@ua.es

Juan Francisco Álvarez Herrero. Doctor en Tecnología Educativa por la Universitat Rovira i Virgili. Profesor asociado del Departamento de Didáctica General y Didácticas Específicas de la Universidad de Alicante y miembro del Grupo de Investigación: EDUTIC-ADEI (Educación y Tecnologías de la Información y Comunicación-Atención a la Diversidad. Escuela Inclusiva) de la misma universidad. $\mathrm{Su}$ investigación se interesa especialmente por la implementación de pedagogías emergentes y metodologías activas, la competencia digital, los Entornos personales de Aprendizaje y las Redes Sociales en el mundo educativo.

E-mail: juanfran.alvarez@ua.es

Dirección:

Facultad de Educación

Universidad de Alicante

Campus Sant Vicent del Raspeig

03690-Sant Vicent del Raspeig (España)

Fecha de recepción del artículo: 21/12/2018

Fecha de aceptación del artículo: 23/01/2019

Fecha de aprobación para maquetación: 31/03/2019 